\title{
A CASE OF RIGHT SIDED \\ STRANGULATED OBTURATOR HERNIA: AN \\ EXTREMELY RARE TYPE \\ OF HERNIA MANAGED
}

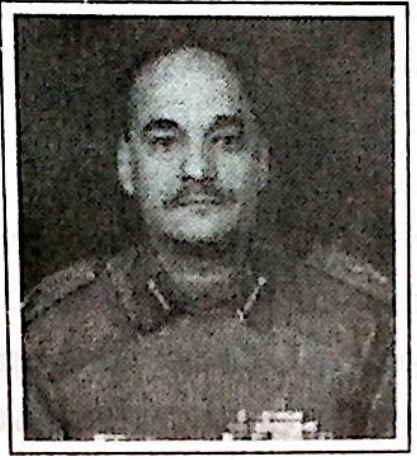

\section{Abstract}

Obturator hernia is one of the rarest forms of hernias. Most surgeons will see no more than one or perhaps two in an entire career. Approximately $0.5 \%$ of the mechanical intestinal obstruction is caused by strangulated obturator hernia. Our experience in one such case is reported.

Key Words: obturator hernia, leucocytosis, obturator membrane

\section{Introduction}

Obturator hernias are more common in elderly female. These present usually as acute intestinal obstruction and are diagnosed at the time of operation. The sac usually contains strangulated bowel loops. It should be considered as possible diagnosis when an elderly thin built patient presents with acute intestinal obstruction of unknown cause. Recently pelvic CT scan has been used to diagnose it preoperatively in suspected cases. However, preoperative diagnosis does not influence the outcome. Early surgical
ES Col. Dr. B.K. Hamal, MS, FICS

Consultant Surgeon

ES Dr. Ganesh Thapa, MS Resident

Sr B.G. Karmacharya, MS Resident

intervention should be carried out as there is increased risk of strangulation.

\section{Case report}

Previously healthy 72 years old female presented with abdominal pain of 7 days duration. Pain was colicky in nature, associated with repeated vomiting and constipation. There was no other significant past illnesses.

On examination, she was thin built, tachycardic and dehydrated. Abdominal examination revealed distended abdomen with visible peristalsis, generalized tenderness and increased bowel sounds. Investigations revealed leucocytosis. Other routine hematological and biochemical exams were normal. $X$-ray of abdomen showed multiple air fluid levels. With diagnosis of acute intestinal obstruction, she was resuscitated and prepared for emergency laparotomy. There was strangulated right sided obturator hernia containing about $3 \mathrm{cms}$ long, Richter's type segment of small intestine. End to end resection and side to side anastomosis was performed. The postoperative period was uneventful. 


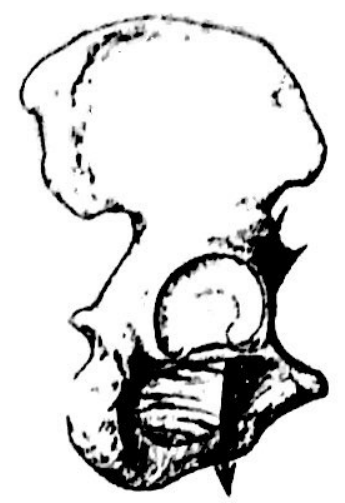

Obturator canal

\section{Discussion}

Obturator hernia is one of the rarest forms of hernia. Most surgeons will see no more than one or perhaps two in an entire career'. The hernial sac passes through the obturator canal which is bounded above superior pubic ramus and below by the sharp free upper border of the obturator membrane. The canal is about three cms long and runs downwards, forwards and medially. The obturator vessels and nerve pass from the abdomen to the medial aspect of thigh. The sac contains usually the small intestinal loops but rarely appendix, ovaries, Meckel's diverticulum, ometnum, bladder and fallopian tube. Approximately $0.5 \%$ of mechanical intestinal obstruction is caused by obturator hernia. The male female ratio is 1:6. High incidence in female is due to broader pelvis, wide obturator canal and pregnancy. The age of patients in reported cases varies from 12 to 93 years but most cases occur during the 7 th and 8 th decades.

There are four cardinal features of obturator hernia, although they appear together in less than $5 \%$. They are acute intestinal obstruction( $88 \%$, with gangrenous bowel in 60-75\%)2, Howship-Romberg sign(55\%), repeated attacks of intestinal obstruction that pass spontaneously $(30 \%)$ and palpable mass or lump in the medial aspect of the thigh at the origin of adductor muscles below pectineus $(20 \%)$. In case of strangulated obturator hernia there might be ecchymosis in the medial part of the groin below the inguinal ligament and tender mass in the obturator area, felt laterally in the PV or PR examination.

Early diagnosis of obturator hernia is relatively difficult ${ }^{3}$.None of the investigations has been diagnostic for obturator hernia. Recently pelvic CT scan has been shown to provide diagnosis in suspected cases and may be indicated in elderly patients with mechanical intestinal obstruction of unknown origin. Nakayama T et $\mathrm{l}^{4}$ has reported $100 \%$ sensitivity of pelvic CT in diagnosing obturator hernias preoperatively. However, the correct preoperative diagnosis does not appearinfluence the outcome; the survival being determined by early surgical intervention.

All obturator hernias should be operated on soon after the diagnosis as the risk of strangulation is high. After dealing with the contents, the hernial orifice is closed with simple suturing, using mesh or using broad ligament to close the internal opening.

\section{References}

1. Zinner M.J., Schwarz S.I., Ellis H.eds. (1998) Maingot's Abdominal Operations, 10th ed.Appleton-Lange,

2. Mann C, Russel R.C.G., Williams N.S.(eds)(1995) Bailey and Love's Short Practice of Surgery,22nd edn, Chapman and Hall, London

3. XU Qingxiang et al. Strangulated Obturator hernias: Report of 2 cases Chin Med J 2002; 115(9): 1420-21

4. NakayamaT et al.Diagnosis and treatment of obturator hernia. Keio J Med 2002 Sep; 51(3) 129-32 